\title{
Atrophy and hypertrophy signalling of the quadriceps and diaphragm in COPD
}

\author{
Mariève Doucet, ${ }^{1}$ Annie Dubé, ${ }^{1}$ Denis R Joanisse, ${ }^{1,2}$ Richard Debigaré, ${ }^{1}$ \\ Annie Michaud, ${ }^{1}$ Marie-Ėve Paré, ${ }^{1}$ Rosaire Vaillancourt, ${ }^{1}$ Éric Fréchette, ${ }^{1}$ \\ François Maltais ${ }^{1}$
}

\begin{abstract}
- Additional data are published online only. To view this file, please visit the journal online (http://thorax.bmj.com).

${ }^{1}$ Centre de recherche, Institut Universitaire de Cardiologie et de Pneumologie de Québec, Université Laval, Ouébec, Canada

${ }^{2}$ Division de Kinésiologie, Université Laval, Québec, Canada
\end{abstract}

\section{Correspondence to}

Dr François Maltais, Centre de Pneumologie, Institut Universitaire de Cardiologie et de Pneumologie de Québec, 2725 Chemin Ste-Foy, Québec, QC, G1V 4G5, Canada; francois.maltais@med.ulaval.ca

Received 18 December 2009 Accepted 29 June 2010

\section{ABSTRACT}

Background Factors involved in the regulation of muscle mass in chronic obstructive pulmonary disease (COPD) are still poorly understood. Comparing the signalisation involved in muscle mass regulation between two muscles with different levels of activation within the same subjects is an interesting strategy to tease out the impact of local (muscle activity) versus systemic factors in the regulation of muscle mass. A study was undertaken to measure and compare the protein levels of p-AKT, AKT, Atrogin-1, p-p70S6K, p-4E-BP1, p-GSK3 $\beta$ as well as the mRNA expression of Atrogin-1, MuRF1 and FoxO-1 in the quadriceps and the diaphragm of 12 patients with COPD and 7 controls with normal lung function.

Methods Diaphragm biopsies were obtained during thoracic surgery and quadriceps samples were obtained from needle biopsies. Protein content and mRNA expression were measured by western blot and quantitative PCR, respectively.

Results Increased mRNA expressions of Atrogin-1, MuRF1 and Fox0-1 were found in the quadriceps compared with the diaphragm only in patients with COPD. The quadriceps/diaphragm ratio for MuRF1 was higher in COPD. The protein level of p-p70S6K was decreased in the quadriceps compared with the diaphragm in patients with COPD. The quadriceps/ diaphragm ratios of $p$-p70S6K and p-GSK3 $\beta$ were lower in patients with COPD than in controls.

Conclusions These results indicate a greater susceptibility to a catabolic/anabolic imbalance favouring muscle atrophy in the quadriceps compared with the diaphragm in patients with COPD. The balance between the atrophy and hypertrophy signalling is inhomogeneous between respiratory and lower limb muscles, suggesting that local factors are likely to be involved in the regulation of muscle mass in COPD.

\section{INTRODUCTION}

Limb muscle dysfunction affects functional status, ${ }^{1}$ quality of life $e^{2}$ and survival in chronic obstructive pulmonary disease (COPD). ${ }^{3}$ Inspiratory muscle dysfunction is associated with negative clinical consequences such as dyspnoea, hypercapnic respiratory failure ${ }^{4}$ and even premature mortality. ${ }^{5}$ Among the different facets of skeletal muscle dysfunction, the regulation of muscle mass has attracted the attention of several research teams because of the clinical relevance of muscle wasting in COPD and other chronic diseases.

The maintenance of muscle mass may be compromised in a variety of clinical situations. Ageing, hypoxaemia, systemic inflammation (chronic or occurring during acute bursts in relation to COPD exacerbations) ${ }^{6}$ nutritional imbalance and oxidative stress may all threaten the preservation of muscle mass. ${ }^{7-9}$ Decreased muscle activation may also create an imbalance in the regulation of muscle mass that promotes catabolism ${ }^{10}{ }^{11}$ at the expense of anabolism. ${ }^{12}$

These observations on the potential role of muscle activation as a cause of muscle dysfunction and wasting are relevant to COPD as chronic inactivity reduces the degree of quadriceps activation, even early in the disease process, ${ }^{13}$ while increased work of breathing results in a chronically activated diaphragm. Based on this divergent level of muscle activation between limb and respiratory muscles, one would predict that the pathways involved in the regulation of the quadriceps and diaphragmatic muscle mass should be regulated differentially in patients with COPD, with the former muscle expected to be more susceptible to catabolism and the latter exhibiting a tendency towards increased anabolism.

We recently explored atrophy/hypertrophy signalling pathways involved in the regulation of quadriceps muscle mass in wasted patients with COPD. ${ }^{14}$ As we predicted, this study showed an activation of the ubiquitin-proteasome pathway consistent with a catabolic state in the quadriceps of patients with COPD compared with controls with normal lung function. ${ }^{14}$ Similar findings have been reported by other investigators. ${ }^{15}$ Our investigation also revealed, in the same individuals, an overexpression of the muscle insulin-like growth factor-1 (IGF-1) hypertrophy signalling pathways that is not consistent with muscle atrophy and could represent a failed attempt to restore muscle mass. $^{14}$

The regulation of diaphragmatic muscle mass is another active field of investigation. In apparent discrepancy with our hypothesis, an imbalance in the catabolic/anabolic status favouring catabolism has also been reported in patients with COPD. ${ }^{16-18}$ These findings were somewhat unexpected because the continuous training stimulus to which the COPD diaphragm is exposed should trigger anabolism. ${ }^{19-22}$

Activation of the ubiquitin-proteasome pathway in the quadriceps and the diaphragm does not mean that these two muscles are equally susceptible to catabolism. One difficulty in interpreting the current literature is that the comparative assessment of the quadriceps and diaphragm signalling 
pathways in COPD is based on comparisons of muscle samples that do not originate from the same subjects. This makes it impossible to rule out the potential role of confounders such as nutritional status, smoking status, exposure to corticosteroids and hypoxaemia that may influence the regulation of muscle mass. This concern can only be addressed by comparing the two muscle groups within the same individuals. Thus, using this approach, our aim was to measure and compare the mRNA and protein levels of the ubiquitin-proteasome and the IGF-1/Akt pathways in both muscle groups within the same individuals.

The present study was first designed to test the hypothesis that the ubiquitin-proteasome and the IGF-1/AKT pathways would be differentially regulated in the quadriceps muscle compared with the diaphragm in patients with COPD. Quadriceps and diaphragm biopsies obtained from the same patients showed a clear differential regulation of the biochemical pathways of interest in the vastus lateralis and the diaphragm in COPD.

These results naturally led to the question of whether this differential muscle mass regulation between the quadriceps and the diaphragm was specific to COPD or a normal phenomenon seen in healthy individuals. To address this, we recruited subjects with normal lung function from whom we obtained quadriceps and diaphragm biopsies. Our second hypothesis was that a differential regulation of the ubiquitin-proteasome and IGF-1/ AKT pathways between the quadriceps and the diaphragm, if present, would be of a smaller magnitude in individuals with normal lung function than in those with COPD.

\section{METHODS}

\section{Subjects}

Twelve patients with COPD undergoing lung resection were recruited. Nutritional status was evaluated with anthropometric parameters and serum albumin levels at the time of the investigation. Patients had not been exposed to systemic corticosteroids during the 2 months preceding their participation in the study and none were receiving long-term oxygen therapy. Seven subjects with normal lung function were also recruited in whom the same inclusion/exclusion used in COPD were applied except for lung function. None of the participating subjects was involved in our previous comparative study between the diaphragm and the quadriceps. ${ }^{22}$ Patients with body weight loss that could be ascribed to cancer were excluded. Further information on study subjects can be found in the online data supplement.

\section{Pulmonary function tests and anthropometric measurements}

Standard pulmonary function tests including spirometry, lung volumes with body plethysmography and transfer factor (diffusion capacity) were obtained according to previously described guidelines ${ }^{23}$ and related to the normal values of Quanjer et al. ${ }^{24}$ Height and weight were measured according to standardised methods. ${ }^{25}$

\section{Muscle biopsies Diaphragm}

Diaphragm biopsies were obtained during a thoracic surgical procedure (thoracoscopy or thoracotomy). ${ }^{22}$ Either the right or the left diaphragm was biopsied, depending on the side of the surgery. Muscle samples were taken from the costal median region of the diaphragm (COPD: right side in 9/12; controls: right side in 4/7). Diaphragm specimens were frozen in liquid nitrogen and stored at $-70^{\circ} \mathrm{C}$ for subsequent analysis.

\section{Quadriceps}

Needle biopsies of the quadriceps performed as routinely done in our laboratory ${ }^{26}$ were obtained within $24 \mathrm{~h}$ of the diaphragm biopsy. Muscle specimens were immediately frozen in liquid nitrogen and stored at $-70^{\circ} \mathrm{C}$ for future analysis.

\section{Skeletal muscle analysis}

\section{Muscle fibre cross-sectional area determination}

The mean muscle fibre cross-sectional area (CSA) was determined for each muscle. Briefly, serial consecutive $5 \mu \mathrm{m}$ cryosections were prepared from frozen muscle samples. The sections were immunohistochemically stained ${ }^{22}$ and all muscle sections were visualised by light microscopy and images digitally captured using Image Pro Plus 4.1 for Windows (MediaCybernetics, Silver Spring, Maryland, USA). The fibre CSA was calculated based on 80 randomly selected fibres for each muscle. ${ }^{27}$

\section{RNA extraction and quantitative PCR}

Total RNA extraction was performed from approximately $15 \mathrm{mg}$ of muscle (TRIzol Reagent, Invitrogen, Carlsbad, California, USA); $1 \mu \mathrm{g}$ of RNA was reverse transcripted to cDNA using Quantitect Reverse Transcription Kit (Qiagen Inc, Valencia, California, USA). Real-time PCR was performed in an Opticon 2 (MJ Research, Waltham, Massachusetts, USA) using Quantitect SYBR Green PCR Kit (Qiagen Inc). Further details on the PCR are provided in the online data supplement.

\section{Protein extraction and western blotting}

Cytoplasmic protein extraction was performed with approximately $30 \mathrm{mg}$ of muscle using a commercial kit according to the manufacturer's protocol (NE-PER; Pierce Biotechnology, Rockford, Maryland, USA) for subsequent western blot analysis. The methodologies are described in further detail in the online data supplement.

Table 1 Patient characteristics

\begin{tabular}{|c|c|c|c|}
\hline & $\begin{array}{l}\text { Patients with } \\
\text { COPD }(n=12)\end{array}$ & $\begin{array}{l}\text { Control subjects } \\
(n=7)\end{array}$ & p Value \\
\hline Male/female & $8 / 4$ & $2 / 5$ & 0.1698 \\
\hline Age (years) & $60 \pm 7$ & $60 \pm 11$ & 0.9977 \\
\hline Weight (kg) & $70 \pm 22$ & $71 \pm 12$ & 0.9245 \\
\hline BMI $\left(\mathrm{kg} / \mathrm{m}^{2}\right)$ & $25 \pm 6$ & $27 \pm 3$ & 0.4466 \\
\hline $\mathrm{FEV}_{1}$ (I) & $1.42 \pm 0.70$ & $2.42 \pm 0.80$ & 0.0072 \\
\hline $\mathrm{FEV}_{1}(\%$ predicted $)$ & $50 \pm 17$ & $103 \pm 6$ & $<0.0001$ \\
\hline $\mathrm{FEV}_{1} / \mathrm{FVC}(\%)$ & $53 \pm 14$ & $74 \pm 2$ & 0.0002 \\
\hline ITGV (\% predicted) & $118 \pm 17$ & $111 \pm 5$ & 0.1750 \\
\hline RV (\% predicted) & $172 \pm 44$ & $109 \pm 18$ & 0.0008 \\
\hline RV/TLC (\%) & $52 \pm 11$ & $37 \pm 6$ & 0.0087 \\
\hline TLco (\% predicted) & $75 \pm 20$ & $101 \pm 22$ & 0.0325 \\
\hline $\mathrm{PaO}_{2}(\mathrm{~mm} \mathrm{Hg})$ & $69.8 \pm 10.7$ & - & - \\
\hline $\mathrm{PaCO}_{2}(\mathrm{~mm} \mathrm{Hg})$ & $39.9 \pm 0.3$ & - & - \\
\hline Albumin $(g / l)$ & $43 \pm 3$ & $43 \pm 1$ & 0.5773 \\
\hline \multicolumn{4}{|l|}{ Final diagnosis } \\
\hline \multicolumn{4}{|c|}{ Non-small cell lung carcinoma } \\
\hline Stage la & 0 & 3 & \\
\hline Stage $\mathrm{lb}$ & 5 & 1 & \\
\hline Stage IIla & 0 & 1 & \\
\hline Stage IIlb & 1 & 0 & \\
\hline Non-pulmonary cancer & 0 & 1 & \\
\hline Bullous emphysema & 4 & 0 & \\
\hline Subapical blebs & 0 & 1 & \\
\hline Benign pulmonary nodule & 2 & 0 & \\
\hline
\end{tabular}

$\mathrm{BMI}$, body mass index; $\mathrm{FEV}_{1}$, forced expiratory volume in $1 \mathrm{~s} ; \mathrm{FVC}$, forced vital capacity; ITGV, intrathoracic gas volume; $\mathrm{Pac}_{2}, \mathrm{PaO}_{2}$, arterial carbon dioxide and oxygen tensions; $\mathrm{RV}$, residual volume; TLC, total lung capacity; TLCo, transfer factor. 


\section{Statistical analysis}

The results are expressed as mean $\pm \mathrm{SD}$ unless otherwise specified. Between muscle group comparisons were performed using paired Student t tests or $\chi^{2}$ tests for gender distribution. Possible relationships between muscle mRNA and protein levels and pulmonary function were evaluated using Pearson correlations. To highlight the impact of COPD in the differential regulation of the ubiquitin-proteasome and IGF-1/AKT pathways in the two muscle groups, we compared the quadriceps to diaphragm ratio for the expression of the different $\mathrm{mRNA}$ and proteins of interest between subjects with COPD and those with normal lung function using unpaired t tests. The results were considered statistically significant if $\mathrm{p}$ values were $<0.05$.

\section{RESULTS}

\section{Subject characteristics}

On average, patients with COPD had moderate to severe airflow obstruction (table 1). Six patients had stage II disease according to the GOLD classification ${ }^{28}$; the remaining had stage III disease. Body mass index and serum albumin levels were normal in all patients. Thirteen of the 19 study subjects were suspected of having a resectable lung cancer while one subject was diagnosed with carcinoma of the oesophagus. On final analysis, 11 had lung cancer and two patients had a benign tumour, and one patient had oesophageal lymphoma. This last patient was kept in the study since there was no extension of the lymphoma outside the thorax. One patient with normal lung function underwent surgery for recurrent pneumothoraces related to subapical blebs and four patients with COPD had a bullectomy. Patients with cancer did not differ in terms of their body mass index and clinical status from those with a benign condition. Mean quadriceps and diaphragm muscle fibre CSA were similar in both groups $\left(5388 \pm 2043 \mu \mathrm{m}^{2}\right.$ and $4968 \pm 1504 \mu \mathrm{m}^{2}$ for the quadriceps and diaphragm in patients with COPD; $4287 \pm 573 \mu \mathrm{m}^{2}$ and $4319 \pm 1383 \mu \mathrm{m}^{2}$ for the quadriceps and diaphragm in controls). There were no statistically significant between-group differences in quadriceps or diaphragm muscle fibre CSA.

\section{Muscle atrophy and hypertrophy signalling in subjects with COPD}

The mRNA expression of Atrogin-1, MuRF1 and FoxO-1 was significantly increased in the quadriceps compared with the diaphragm in patients with COPD (figure $1 \mathrm{~A}-\mathrm{C}$ ). A significant positive correlation was observed between Atrogin-1 and MuRF1 mRNA expression in the quadriceps $\left(r^{2}=0.78, p<0.001\right)$

\section{COPD}

A

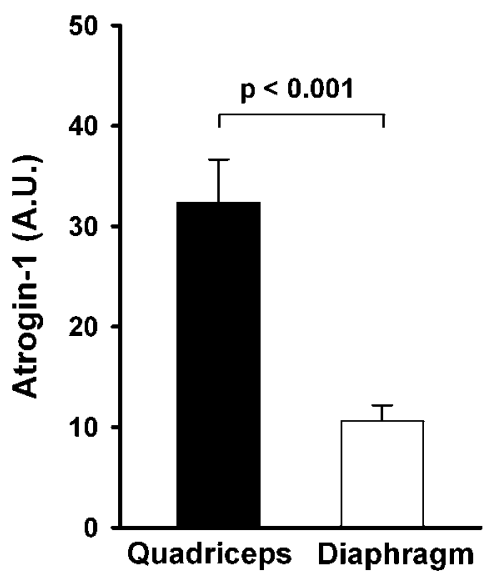

B

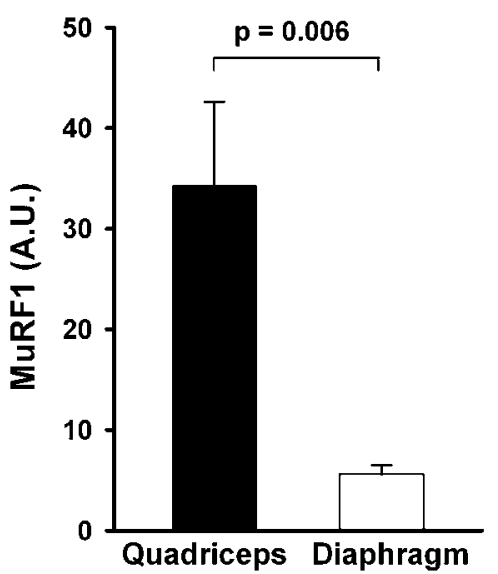

C

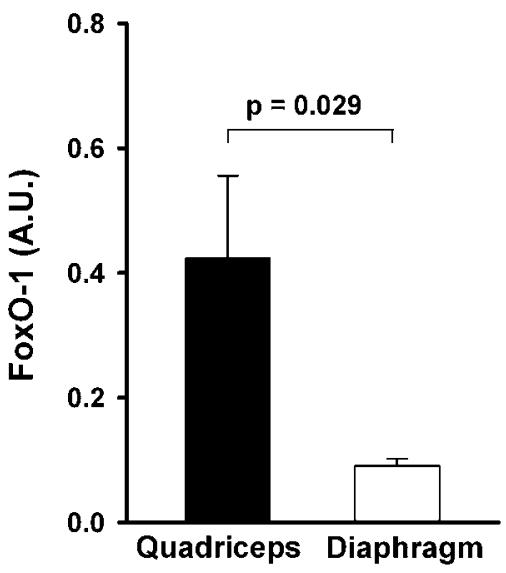

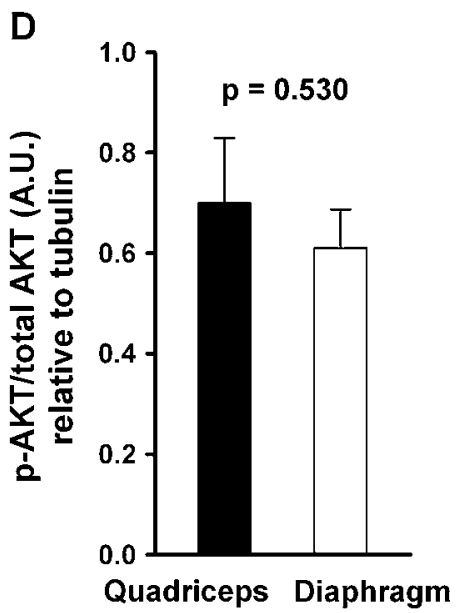
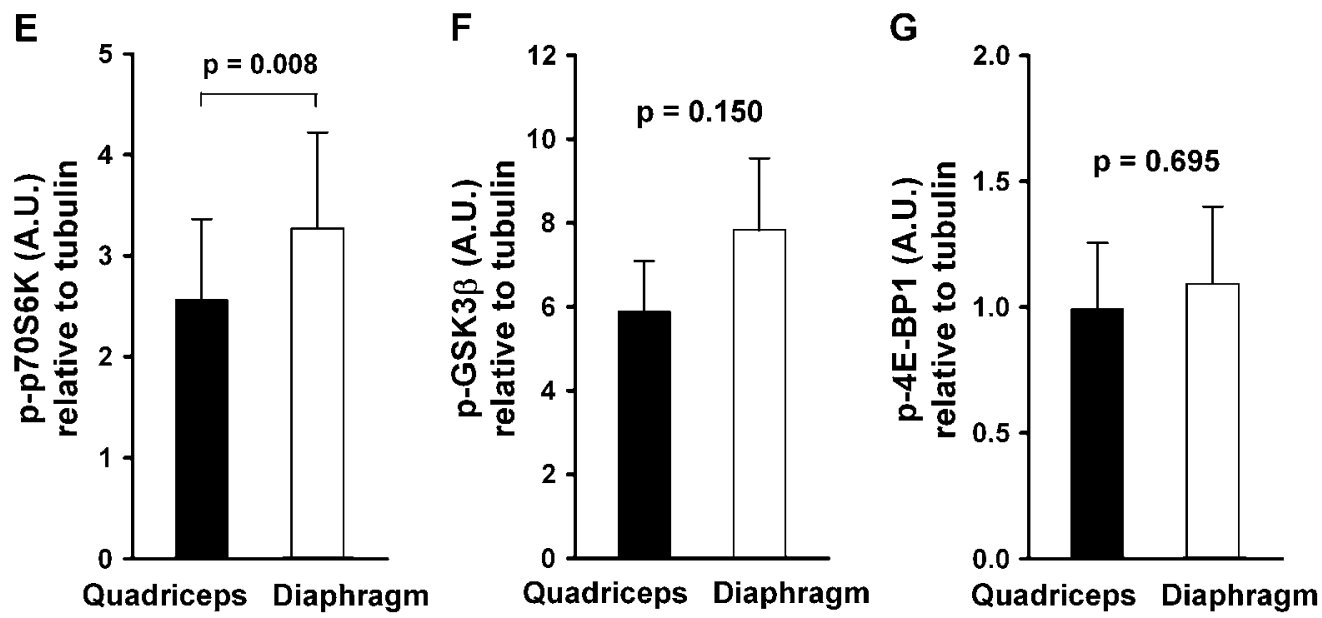

Figure 1 mRNA expression of (A) Atrogin-1, (B) MuRF1 and (C) Fox0-1 and protein content of (D) phosphorylated AKT/total AKT and the phosphorylated form of (E) p70S6K, (F) GSK3 $\beta$ and (G) 4E-BP1 in the quadriceps and diaphragm of patients with chronic obstructive pulmonary disease (COPD). Data are presented in arbitrary units (AU). Values are mean \pm SEM. 
and in the diaphragm $\left(\mathrm{r}^{2}=0.60, \mathrm{p}=0.003\right)$. In the quadriceps, positive correlations were found between Atrogin-1 and FoxO-1 $\left(\mathrm{r}^{2}=0.56, \mathrm{p}=0.005\right)$ and between MuRF1 and FoxO-1 $\left(\mathrm{r}^{2}=0.45\right.$, $\mathrm{p}=0.016) \mathrm{mRNA}$ levels. Atrogin-1 protein expression was similar between the quadriceps and the diaphragm (data not shown).

There were no significant differences in the protein levels of phosphorylated-AKT and the phosphorylated-AKT/total AKT ratio (figure $1 \mathrm{D}$ ). p70S6K was significantly downregulated in its phosphorylated state in the quadriceps compared with the diaphragm in patients with COPD (figure 1E). There were no significant differences in phosphorylated-GSK3 $\beta$ and phosphorylated-4E-BP1 between the two muscles (figure 1F,G).

\section{Muscle atrophy and hypertrophy signalling in subjects with normal lung function}

In contrast to patients with COPD, the mRNA expression of Atrogin-1, MuRF1 and FoxO-1 was similar in the quadriceps and the diaphragm of controls (figure $2 \mathrm{~A}-\mathrm{C}$ ). There were also no significant differences in the levels of phosphorylated-AKT and the phosphorylated-AKT/total AKT ratio or in phosphorylatedp70S6K, -GSK3 $\beta$ and -4E-BP1 between the two muscles in control subjects (figure $2 \mathrm{D}-\mathrm{G}$ ).
Quadriceps to diaphragm ratio of the ubiquitin-proteasome and IGF-1/AKT pathways

Muscle samples of the two study groups were analysed sequentially. For this reason and because material from patients with COPD was insufficient to repeat the experiments, it was not possible to make direct between-group comparisons of the regulation pathways of the quadriceps and the diaphragm. To further assess whether the differential expression of the ubiquitin-proteasome and IGF-1/AKT pathways was specific to COPD, we compared the quadriceps to diaphragm ratio of the different $\mathrm{mRNA}$ and proteins of interest between subjects with COPD and controls. The quadriceps to diaphragm ratio for Atrogin-1 and MuRF-1 mRNA was higher in subjects with COPD than in those with normal lung function, indicating a greater susceptibility to protein degradation in the quadriceps than in the diaphragm in subjects with COPD (figure 3A,B). Conversely, the quadriceps to diaphragm ratio for two proteins involved in hypertrophic signalling (p70S6K and GSK3 $\beta$ ) was lower in subjects with COPD, indicating less hypertrophic stimulation in the quadriceps than in the diaphragm in COPD than in individuals with normal lung function (figure $3 \mathrm{E}, \mathrm{F}$ ).

\section{Controls}

A

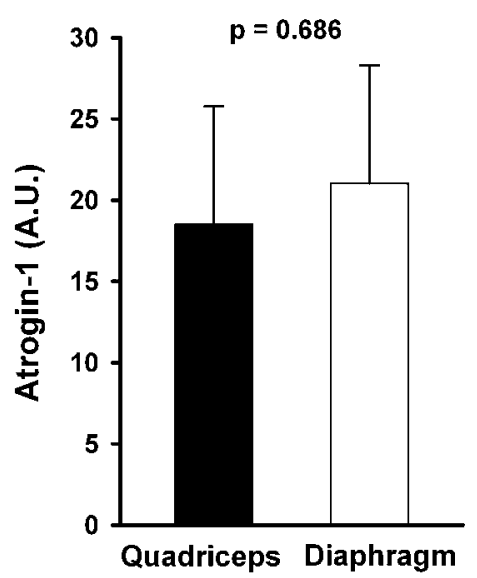

B

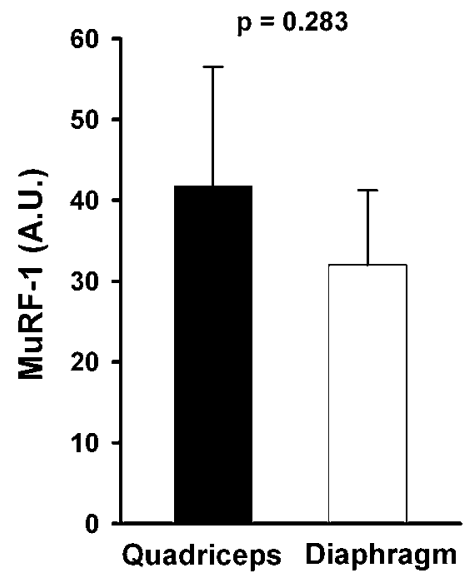

C

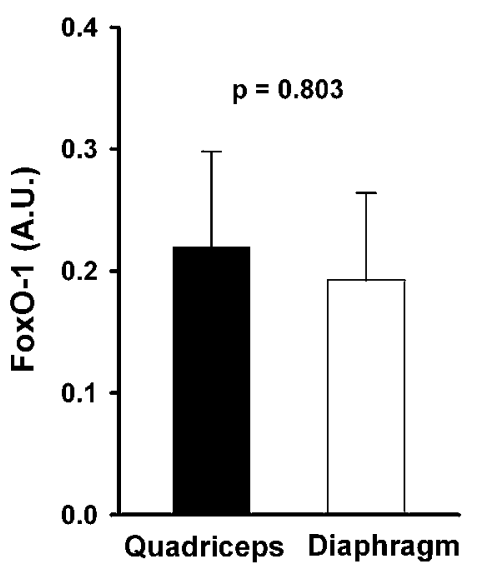

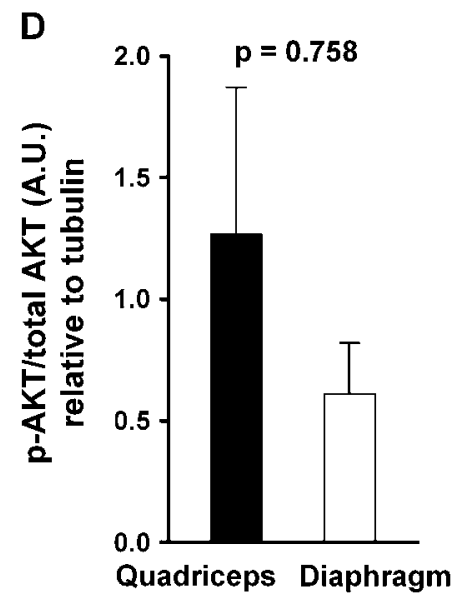
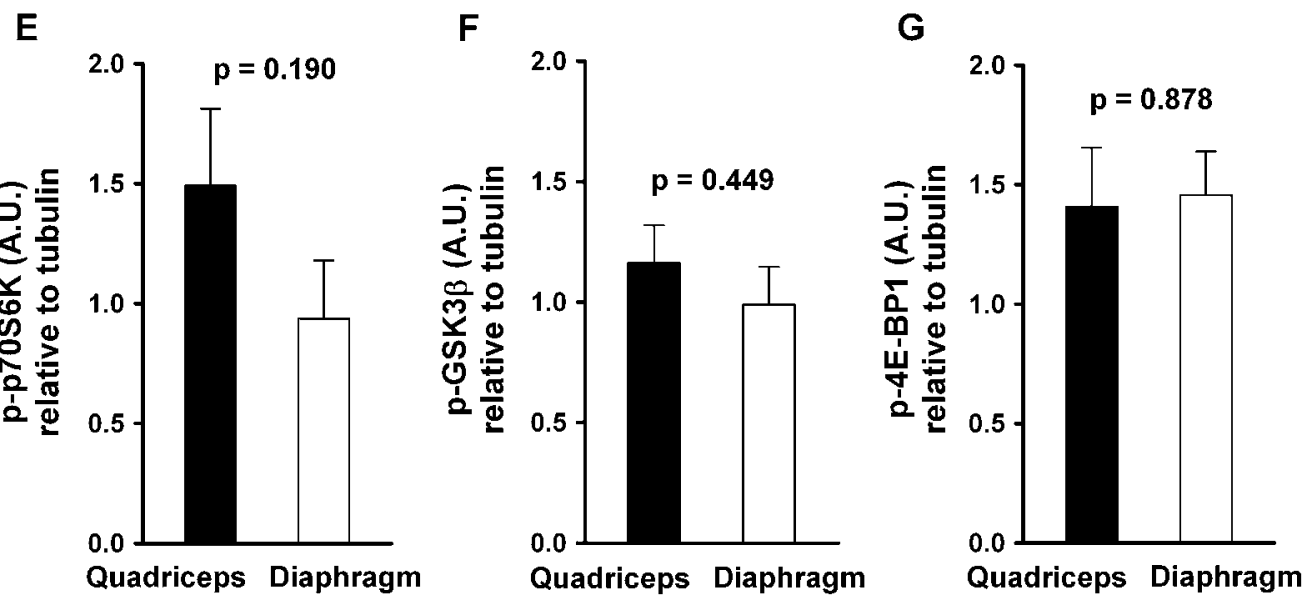

Figure 2 mRNA expression of (A) Atrogin-1, (B) MuRF1 and (C) Fox0-1 and protein content of (D) phosphorylated AKT/total AKT and the phosphorylated form of (E) p70S6K, (F) GSK3 $\beta$ and (G) 4E-BP1 in the quadriceps and diaphragm of subjects with normal lung function. Data are presented in arbitrary units (AU). Values are mean \pm SEM. 
A

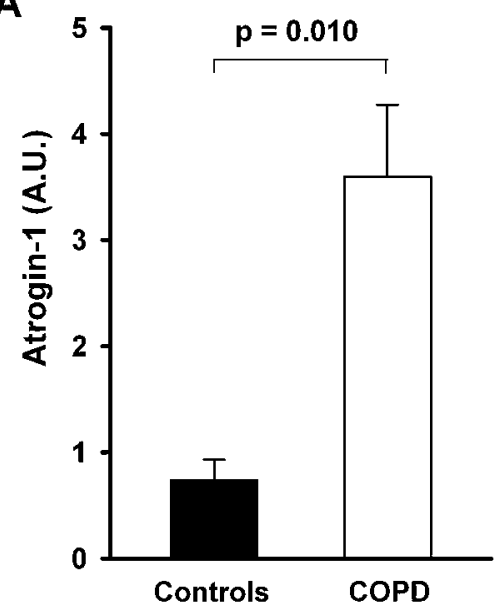

Quadriceps to diaphragm ratio

B

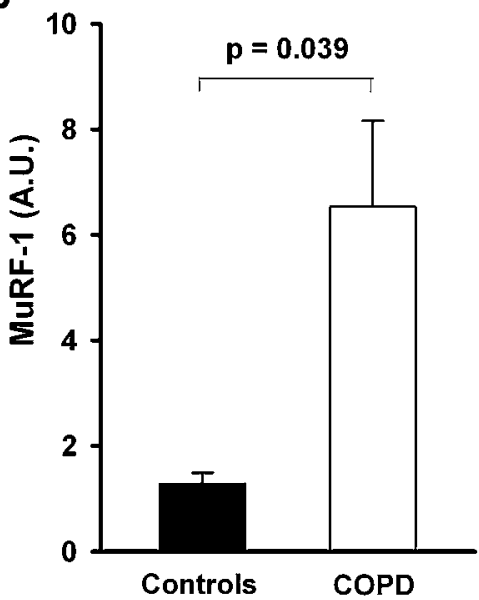

C

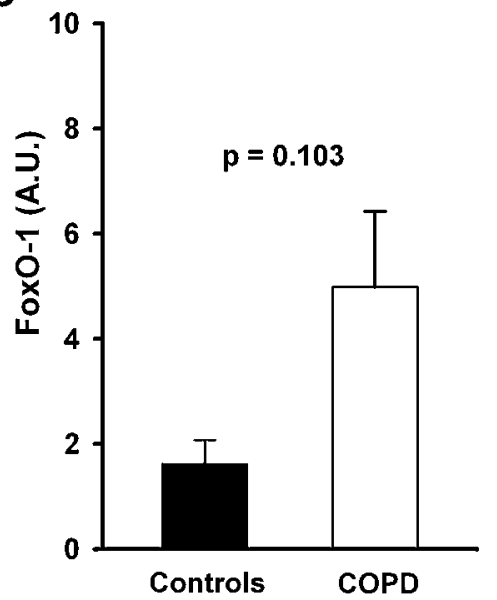

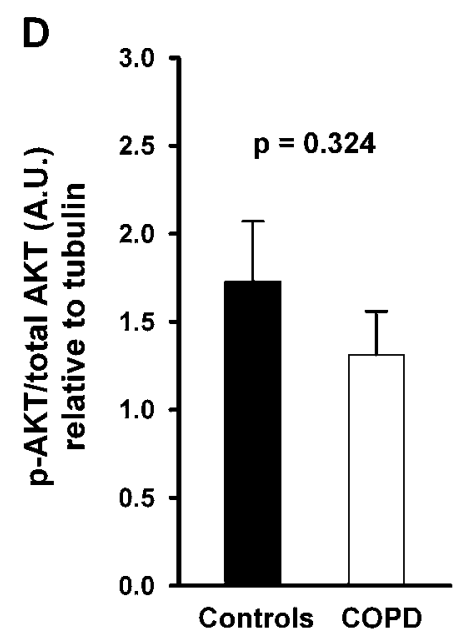

E

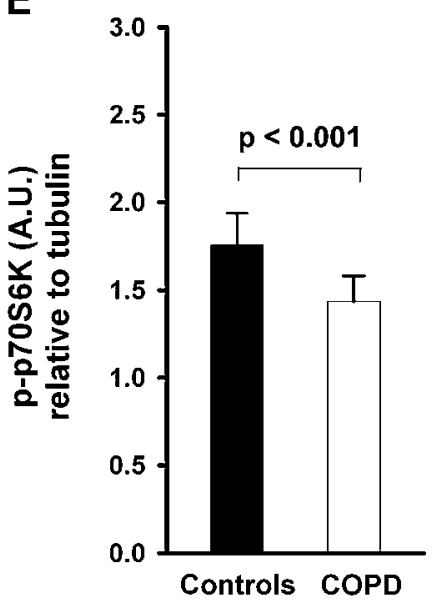

$\mathbf{F}$

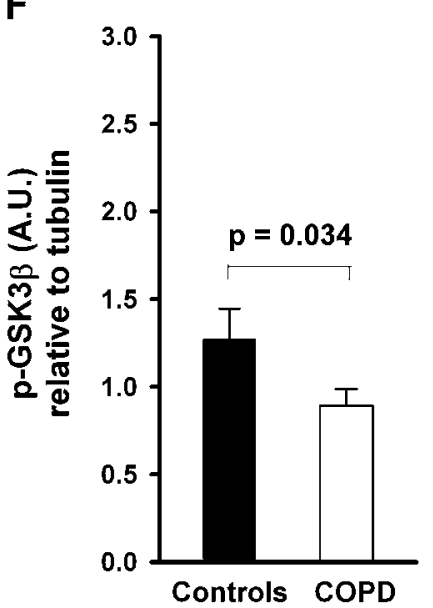

G

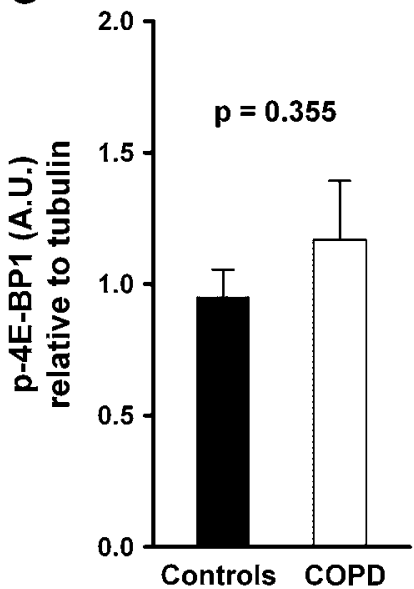

Figure 3 Quadriceps to diaphragm ratio for (A) Atrogin-1, (B) MuRF1 and (C) Fox0-1 mRNA expression and protein content of (D) phosphorylated AKT/total AKT and the phosphorylated form of (E) p70S6K, (F) GSK3 $\beta$ and (G) 4E-BP1 in subjects with normal lung function and those with chronic obstructive pulmonary disease (COPD). Values are mean \pm SEM.

\section{Relationships between diaphragm signalisation and pulmonary function}

The phosphorylated-4E-BP1 diaphragm protein content did not correlate with forced expiratory volume in $1 \mathrm{~s}\left(\mathrm{FEV}_{1}\right)$ percentage predicted (figure 4A). As shown in figure 4B, there was a positive relationship between diaphragm phosphorylated-4E-BP1 protein content and $\mathrm{FEV}_{1} / \mathrm{FVC}$ ratio $\left(\mathrm{FEV}_{1} / \mathrm{FVC} \%: \mathrm{r}^{2}=0.52, \mathrm{p}=0.013\right)$. Phosphorylated-4E-BP1 protein content was inversely correlated with intrathoracic gas volume (ITGV), a surrogate of functional residual capacity, and residual volume (RV) (ITGV\% predicted: $r^{2}=0.68, p=0.003 ; R V \%$ predicted: $r^{2}=0.53, p=0.017$; figure $4 \mathrm{C}, \mathrm{D})$. No other correlation with phosphorylated forms of AKT, p70S6K or GSK3 $\beta$ protein content was found with pulmonary function. There were no statistically significant correlations between the E3-ligases and indices of lung function.

\section{DISCUSSION}

This study provides a thorough investigation of key signalling pathways involved in the regulation of muscle mass in patients with COPD. A major feature of this study was the opportunity to investigate these pathways in two different muscles taken from the same individuals. Comparison with the diaphragm demonstrated greater catabolism and concomitant lower anabolism signalling in the quadriceps in patients with COPD.
Although the study design did not allow a direct comparison of the regulating pathways of the two muscle groups between the two study groups, the data obtained in control subjects were useful in showing that this differential regulation of muscle mass between the quadriceps and the diaphragm was a specific feature of COPD, which suggests that this does not simply reflect a physiological situation but is part of the skeletal muscle adaptation seen in COPD.

The present results support an increased activation of the ubiquitin-proteasome pathway in the COPD quadriceps compared with the diaphragm, as indicated by the increased expression of Atrogin-1 and MuRF1 mRNA. Our data also suggest a role for FoxO-1 in the transcriptional upregulation of these two muscle-specific E3-ligases. In spite of similar AKT protein content, a decrease in the levels of the active form of p70S6K was also found in the COPD quadriceps and could be interpreted as a suboptimal anabolic signal in this muscle. Together these results suggest that the balance between atrophy and hypertrophy signalling is not homogeneous between respiratory and lower limb muscles, the latter muscles being more susceptible to catabolism. An important finding was that this imbalance towards greater susceptibility to catabolism in the quadriceps was not seen in individuals with normal lung function, suggesting that the observations made in subjects with 
Figure 4 Correlation analyses between the phosphorylated form of $4 \mathrm{E}-\mathrm{BP} 1$ protein content in the diaphragm and indices of pulmonary function. The phosphorylated 4E-BP1 protein content did not correlate with (A) forced expiratory volume in $1 \mathrm{~s}$ $\left(\mathrm{FEV}_{1}\right)$ percentage predicted but was positively correlated with $(B)$ the ratio of $\mathrm{FEV}_{1}$ to forced vital capacity $\left(\mathrm{FEV}_{1}\right)$ FVC). Statistically significant inverse correlations were found between phosphorylated 4E-BP1 protein content and (C) intrathoracic gas volume (ITGV) percentage predicted and (D) residual volume (RV) percentage predicted. Protein contents are presented in arbitrary units (AU).
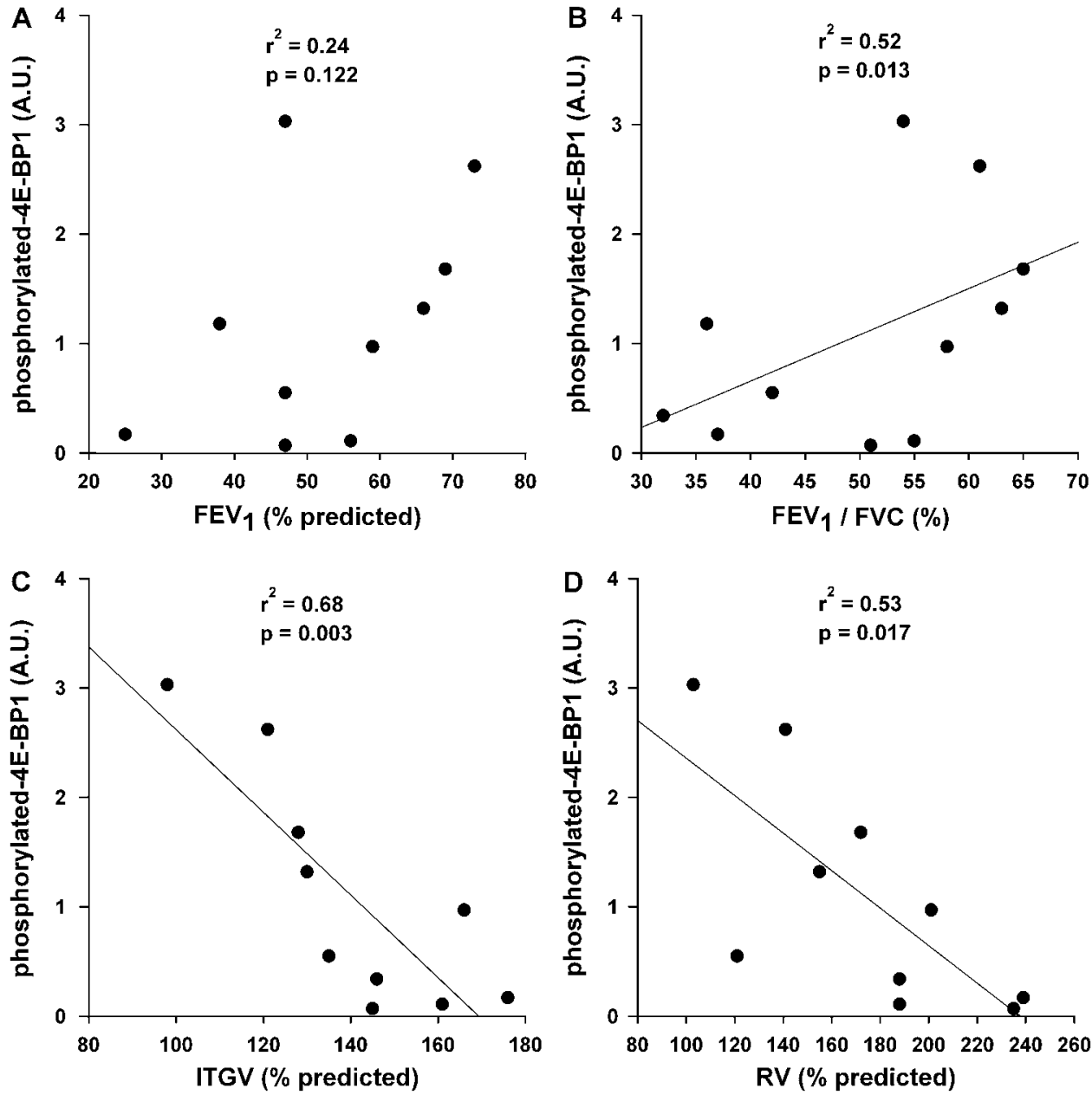

COPD were driven by the underlying pathological process. Although the mechanistic explanation underlying the differential regulation in muscle mass between the COPD quadriceps and diaphragm could not be explored, one likely candidate is a larger difference in activity levels between these two muscles in subjects with COPD than in individuals with normal lung function

Although the level of muscle activation was not measured in this study, physical inactivity is a common feature of COPD which is already present early in the disease process. ${ }^{13} 29$ Conversely, the diaphragm in COPD faces a chronic increase in work load that acts as a continuous source of muscle stimulation. ${ }^{30}$ Mechanical unloading in human models initiates muscle protein breakdown that is mediated via activation of the proteasome components. ${ }^{10} 11{ }^{31}$ Also, the extent of muscle atrophy and upregulation of the ubiquitin-proteasome system within a given muscle is tightly related to the magnitude of the reduction in muscle activation. ${ }^{32}$ These results are consistent with the notion that the low level of activity of the quadriceps could favour the signalling of muscle protein breakdown in patients with COPD

Other interesting findings that support the involvement of local factors in the regulation of muscle mass in COPD are the negative correlations between phosphorylated-4E-BP1 protein content and lung hyperinflation indices. These results suggest that decreased protein synthesis might be triggered by chronic diaphragm shortening. ${ }^{33}$ These muscle adaptations in COPD may promote the loss of contractile proteins, perhaps related to the loss and/or shortening of diaphragm sarcomeres which occurs as part of the adaptation to chronic hyperinflation. ${ }^{30}$ Increased activation of the ubiquitin-proteasome pathway has been reported in the diaphragm of patients with COPD, ${ }^{17}{ }^{18}$ while increased apoptosis and altered regeneration capacity of the diaphragm has been found in emphysematous hamsters. ${ }^{34}$ These data are thus consistent with the notion that COPD may predispose to the loss of diaphragmatic muscle mass although, as suggested by our results, to a lesser extent than the quadriceps.

A possible contribution of systemic factors in the expression of the anabolic/catabolic regulation pathways in the quadriceps and the diaphragm cannot be excluded because these two muscles may react differently to the presence of systemic factors such as inflammatory mediators, corticosteroids and hypoxaemia in their external milieu. Although less likely, the possibility also exists that intrinsic alterations of the quadriceps muscle tissue itself may render it more susceptible to catabolism than the diaphragm.

We found a parallel upregulation in Atrogin-1 and MuRF1 in the quadriceps and the diaphragm in patients with COPD. Although a coordinate expression of these two E3-ligases is often seen in models of atrophy, ${ }^{35}$ this is not always the case. Atrogin- 1 but not MurF1 has been reported to be upregulated in the COPD diaphragm. ${ }^{17}{ }^{18}$ In one study, ${ }^{18} 60 \%$ of the COPD study population was exposed to systemic corticosteroids. This is relevant when considering that Atrogin-1 might be more responsive to corticosteroids than MuRF1. ${ }^{36}$ Thus, discrepancies in the expression pattern of the E3-ligases between studies could be explained by differences in study population and in the specific models of atrophy under study. 
In the present study, an upregulation of FoxO-1 mRNA was found in the quadriceps compared with the diaphragm of patients with COPD. Moreover, the positive associations of FoxO-1 with both E3-ligases support a transcriptional role for FoxO-1 in the regulation of the ubiquitin-proteasome pathway in the quadriceps. In addition to being a negative regulator of muscle mass, FoxO-1 also appears to downregulate several type I fibre-related genes as demonstrated in transgenic mice with muscle-specific overexpression of FoxO-1. ${ }^{37}$ We therefore speculate that FoxO-1 may be involved in the regulation of limb muscle mass and fibre-type expression in patients with COPD. ${ }^{33}$

\section{Potential implications of the findings}

Although there was clear differential expression of the atrophy and hypertrophy signalling pathways of the quadriceps and diaphragm in subjects with COPD favouring atrophy in the quadriceps, we could not demonstrate quadriceps muscle fibre atrophy in comparison to the diaphragm in these individuals. The cross-sectional nature of the current study should be appreciated so that it is not possible to make inference about a possible ongoing atrophy process in the quadriceps. Interestingly, perturbations in the biochemical pathways regulating muscle mass precede overt muscle fibre atrophy and weight loss in experimental $^{38}$ and human models of cachexia. ${ }^{39}$ Our results suggest a similar phenomenon in patients with COPD. Only longitudinal studies with serial muscle biopsies and muscle mass measurements could confirm that biochemical events within the muscles antedate muscle atrophy. One potential implication of this discussion is that consideration should be given to early targeting of the biochemical changes responsible for the atrophying process.

\section{Limitations of the study}

A number of methodological considerations and potential limitations need to be taken into account when interpreting our study. First, the study was not designed to compare directly the ubiqutin-proteasome and AkT/IGF-1 pathways of the quadriceps and the diaphragm in subjects with COPD versus healthy controls. Second, there was a tendency towards an imbalance in sex distribution between the two study groups, although this did not reach statistical significance. However, any influence of hormonal status on the results has been minimised because all studied women were postmenopausal. Third, human diaphragm samples can only be obtained in patients scheduled to undergo a surgical procedure. The presence of a lung neoplasm in half of the patients could have, through cancer-related cachexia, influenced muscle molecular signalling. Any potential confounding effects of any systemic effects related to cancer should have been minimised by the fact that the diaphragm and quadriceps were exposed to the same external milieu. Also, patients with body weight loss that could be ascribed to cancer were excluded. Fourth, information on body composition and whether patients with COPD were involved in a progressive body weight loss process are not available owing to the limited access to these patients prior to surgery. This limitation is partially compensated by the measurements in muscle fibre CSA, a parameter that is related to muscle mass. ${ }^{40}$ Last, the ubiquitin-proteasome activity was evaluated only indirectly and mRNA levels alone were measured for FoxO-1 and MuRF1.

\section{Conclusion}

This study highlights potentially key molecular mechanisms involved in muscle atrophy in patients with COPD by the comparison of two muscles with different levels of activity. Evidence of an increase in atrophic signals in the quadriceps compared with the diaphragm in patients with COPD was demonstrated by the upregulation of mRNA expression of Atrogin-1 and MuRF1. This gene upregulation was likely to be mediated by the expression of FoxO-1. On the other hand, decreased expression of $\mathrm{p}-\mathrm{p} 70 \mathrm{~S} 6 \mathrm{~K}$ in the quadriceps would indicate a low anabolic signal in this muscle. Low anabolic signals combined with increased activation of the catabolic pathway would represent an unfavourable situation for the quadriceps, potentially leading to atrophy. Together, these results suggest that the balance between atrophy and hypertrophy signalling is not homogeneous between respiratory and lower limb muscles and that local factors are involved in the regulation of muscle mass in patients with COPD.

Acknowledgements The authors acknowledge the contribution of Audrey Lapierre for his technical assistance, Serge Simard for his statistical assistance and Marthe Bélanger, Christine Racine, Sabrina Biardel and France Simard for their help in accomplishing this study.

Funding Canadien Institutes of Health Research grant MOP-84091. FM holds a GSK/CIHR Research Chair on COPD at Université Laval.

\section{Competing interests None}

Ethics approval This study was conducted with the approval of the Comite d'Éthique de la Recherche, Institut Universitaire de Cardiologie et de Pneumologie de Québec and each patient signed a written informed consent form.

Contributors All authors have contributed to the current work.

Provenance and peer review Not commissioned; externally peer reviewed.

\section{REFERENCES}

1. Schols AM, Mostert R, Soeters PB, et al. Nutritional state and exercise performance in patients with chronic obstructive lung disease. Thorax 1989;44:937-41.

2. Mostert R, Goris A, Weling-Scheepers C, et al. Tissue depletion and health related quality of life in patients with chronic obstructive pulmonary disease. Respir Med 2000;94:859-67

3. Marquis $\mathbf{K}$, Debigaré $\mathrm{R}$, LeBlanc $\mathrm{P}$, et al. Mid-thigh muscle cross-sectional area is a better predictor of mortality than body mass index in patients with COPD. Am J Respir Crit Care Med 2002;166:809-13.

4. Bégin P, Grassino A. Inspiratory muscle dysfunction and chronic hypercapnia in chronic obstructive pulmonary disease. Am Rev Respir Dis 1991;143:905-12.

5. Gray-Donald K, Gibbons L, Shapiro SH, et al. Nutritional status and mortality in chronic obstructive pulmonary disease. Am J Respir Crit Care Med 1996;153:961-6.

6. Crul T, Spruit MA, Gayan-Ramirez G, et al. Markers of inflammation and disuse in vastus lateralis of chronic obstructive pulmonary disease patients. Eur J Clin Invest 2007; 37:897-904.

7. Debigaré R, Maltais $\mathrm{F}$, Côté $\mathrm{CH}$, et al. Profiling of mRNA expression in quadriceps of patients with copd using microarrays. COPD 2007;5:75-84.

8. Radom-Aizik S, Kaminski N, Hayek S, et al. Effects of exercise training on quadriceps muscle gene expression in chronic obstructive pulmonary disease. J Appl Physiol 2007;102:1976-84.

9. Wagner PD. Possible mechanisms underlying the development of cachexia in COPD. Eur Respir J 2008;31:492-501.

10. Reid MB. Response of the ubiquitin-proteasome pathway to changes in muscle activity. Am J Physiol Regul Integr Comp Physiol 2005;288:R1423-31.

11. Zhang $\mathbf{P}$, Chen $X$, Fan M. Signaling mechanisms involved in disuse muscle atrophy Med Hypotheses 2007;69:310-21.

12. Solomon AM, Bouloux PM. Modifying muscle mass-the endocrine perspective. $J$ Endocrinol 2006;191:349-60.

13. Troosters T, Sciurba F, Battaglia S, et al. Physical inactivity in patients with COPD a controlled multi-center pilot-study. Respir Med 2010;104:1005-11.

14. Doucet $\mathbf{M}$, Russell $\mathrm{AP}$, Léger $\mathrm{B}$, et al. Muscle atrophy and hypertrophy signaling in patients with chronic obstructive pulmonary disease. Am J Respir Crit Care Med 2007:176:261-9.

15. Plant PJ, Brooks D, Faughnan M, et al. Cellular markers of muscle atrophy in chronic obstructive pulmonary disease (COPD). Am J Respir Cell Mol Biol 2010;42:461-71.

16. Ottenheijm CA, Heunks LM, Sieck GC, et al. Diaphragm dysfunction in chronic obstructive pulmonary disease. Am J Respir Crit Care Med 2005;172:200-5.

17. Ottenheijm CA, Heunks LM, Li YP, et al. Activation of the ubiquitin-proteasome pathway in the diaphragm in chronic obstructive pulmonary disease. Am J Respir Crit Care Med 2006;174:997-1002.

18. Testelmans D, Crul T, Maes K, et al. Atrophy and hypertrophy signalling in the diaphragm of patients with COPD. Eur Respir J 2010;35:549-56.

19. Similowski T, Yan S, Gauthier AP, et al. Contractile properties of the human diaphragm during chronic hyperinflation. N Engl J Med 1991;325:917-23.

20. Levine S, Kaiser L, Leferovich J, et al. Cellular adaptation in the diaphragm in chronic osbtructive pulmonary disease. N Engl J Med 1997;337:1799-806. 
21. Mercadier JJ, Schwartz K, Schiaffino S, et al. Myosin heavy chain gene expression changes in the diaphragm of patients with chronic lung hyperinflation. Am J Physiol 1998;274:L527-34.

22. Doucet M, Debigaré R, Joanisse DR, et al. Adaptation of the diaphragm and the vastus lateralis muscle in mild to moderate COPD. Eur Respir J 2004;24:971-9.

23. American Thoracic Society. Standards for the diagnosis and care of patients with chronic obstructive pulmonary disease. Am J Respir Crit Care Med 1995;152:S77-120.

24. Quanjer PH, Tammeling GJ, Cotes JE, et al. Lung volumes and forced ventilatory flows. Report working party standardization of lung function tests, European community for steel and coal. Official statement of the European Respiratory Society. Eur Respir J Supp/ 1993;16:5-40.

25. Heymsfield SB, Williams PJ, Shils ME, et al. Nutritional assessment by clinical and biochemical methods. Modern nutrition in health and disease. Philadelphia: Lea \& Febiger, 1994:812-41.

26. Maltais F, Leblanc $P$, Whittom $F$, et al. Oxidative enzyme activities of the vastus lateralis muscle and the functional status in patients with COPD. Thorax 2000;55:848-53.

27. Blomstrand $\mathbf{E}$, Celsing F, Fridén J, et al. How to calculate human muscle fibre areas in biopsy samples-methodological considerations. Acta Physiol Scand 1984;122:545-51.

28. Rabe KF, Hurd S, Anzueto A, et al. Global strategy for the diagnosis, management, and prevention of chronic obstructive pulmonary disease: Gold Executive Summary. Am J Respir Crit Care Med 2007;176:532-55.

29. Watz H, Waschki B, Meyer T, et al. Physical activity in patients with COPD. Eur Respir J 2009;33:262-72.

30. Orozco-Levi M. Structure and function of the respiratory muscles in patients with COPD: impairment or adaptation? Eur Respir J Supp/ 2003:46:41s-51s.
31. Taillandier D, Aurousseau E, Meynial-Denis D, et al. Coordinate activation of lysosomal, $\mathrm{Ca}^{2+}$-activated and ATP-ubiquitin-dependent proteinases in the unweighted rat soleus muscle. Biochem J 1996;316:65-72.

32. Degens H, Alway SE. Control of muscle size during disuse, disease, and aging. Int J Sports Med 2006;27:94-9

33. Caron MA, Debigaré R, Dekhuijzen PN, et al. Comparative assessment of the quadriceps and the diaphragm in patients with copd. J Appl Physiol 2009; 107:952-61.

34. Degens H, Swisher AK, Heijdra YF, et al. Apoptosis and Id2 expression in diaphragm and soleus muscle from the emphysematous hamster. Am J Physiol Regul Integr Comp Physiol 2007;293:R135-44.

35. Bodine SC, Latres $\mathrm{E}$, Baumhueter $\mathrm{S}$, et al. Identification of ubiquitin ligases required for skeletal muscle atrophy. Science 2001;294:1704-8.

36. Cho JE, Fournier M, Da X, et al. Time course expression of foxo transcription factors in skeletal muscle following corticosteroid administration. J Appl Physiol 2010;108:137-45.

37. Kamei Y, Miura S, Suzuki M et al. Skeletal muscle FOX01 (FKHR) transgenic mice have less skeletal muscle mass, down-regulated type I (slow twitch/red muscle) fiber genes, and impaired glycemic control. J Biol Chem 2004;279:41114-23.

38. Sandri M, Sandri C, Gilbert A, et al. Foxo transcription factors induce the atrophyrelated ubiquitin ligase atrogin-1 and cause skeletal muscle atrophy. Cell 2004;117:399-412

39. Bossola M, Muscaritoli M, Costelli $\mathrm{P}$, et al. Increased muscle proteasome activity correlates with disease severity in gastric cancer patients. Ann Surg 2003;237:384-9.

40. Gosker HR, Engelen MP, van Mameren $\mathrm{H}$, et al. Muscle fiber type IIx atrophy is involved in the loss of fat-free mass in chronic obstructive pulmonary disease. Am J Clin Nutr 2002; 76:113-19.

\section{Lung alert}

\section{Effect of mild emphysema on left heart function}

Severe chronic obstructive pulmonary disease is sometimes associated with cor pulmonale, impaired left ventricular filling and reduced left ventricular stroke volume and cardiac output with preserved left ventricular ejection fraction. However, whether similar changes occur in less severe cases of emphysema and airflow obstruction is unknown.

In this study the authors hypothesised that mild emphysema and airflow obstruction were associated with a decrease in left ventricular end-diastolic volume, left ventricular stroke volume and cardiac output. A total of 2816 individuals (13\% current smokers, 38\% former smokers and $49 \%$ never smokers) with normal lung function as well as airflow obstruction and CT evidence of emphysema were included. Patients with restrictive spirometry and clinical cardiovascular disease were excluded. Emphysema was measured quantitatively on cardiac CT scans, airflow obstruction according to ATS and ERS guidelines and left ventricular volume was determined by cardiac MRI.

After multivariate adjustments, significant associations were noted between left ventricular measurements and both percentage emphysema and the ratio of forced expiratory volume in $1 \mathrm{~s}$ to forced vital capacity $\left(\mathrm{FEV}_{1} / \mathrm{FVC}\right)$. An increase in 10 percentage points in percentage emphysema was associated with a $4.1 \mathrm{ml}$ decrease in left ventricular end-diastolic volume, a $2.7 \mathrm{ml}$ decrease in stroke volume and a $0.19 \mathrm{l} / \mathrm{min}$ decrease in cardiac output. A decrease of 10 percentage points in $\mathrm{FEV}_{1} / \mathrm{FVC}$ ratio was associated with decreases of $1.7 \mathrm{ml}$ in left ventricular end-diastolic volume, $1.5 \mathrm{ml}$ in stroke volume and $0.10 \mathrm{l} / \mathrm{min}$ in cardiac output. These associations were even stronger for current smokers. There were no significant associations between left ventricular ejection fraction and percentage emphysema and $\mathrm{FEV}_{1}$ / FVC ratio.

This study suggests that mild emphysema may be an independent and additional risk factor for cardiac disease. This may have implications for the way we screen and manage patients with mild emphysema.

Barr R, Bluemke D, Ahmed F, et al. Percent emphysema, aiflow obstruction, and impaired left ventricular filling. N Engl J Med 2010;362:217-27.

\section{Brendan Mallia-Milanes}

Correspondence to Brendan Mallia-Milanes, ST5, Hereford County Hospital, Hereford, HR1 2ER, UK; bmilanes@doctors.org.uk

Published Online First 29 September 2010

Thorax 2010;65:970. doi:10.1136/thx.2010.145599 